\title{
A Study on Energy-Efficient Protocol in MANET
}

\author{
Taranveer Kaur \\ CEC Landran
}

\author{
Heena \\ Asstt. Professor, IT Deptt., CEC Landran
}

\begin{abstract}
A mobile wireless infrastructure-less network is a collection of wireless mobile nodes dynamically forming a temporary network without the use of any already available centralized administration. Nodes battery lifetime is very limited in adhoc network. In case if the battery power of node exhausted then it leads to several subdivisions in network. Therefore these subdivided nodes make the censorious spot in the network. These critical nodes can exhaust their battery power earlier because of excessive load and processing for data forwarding. The imbalance in generated loads increases the possibility of failure in network nodes, decreases the route lifetime, make partitions in network and decreases route reliability of the MANETs. Due to this, energy consumption issue becomes a vital research topic in wireless infrastructure -less networks. The power aware routing is a most important design technique for MANETs. This review is based on the routing techniques that are based on the minimization of energy usage of individual nodes and many other ways. This review presents various power aware routing mechanisms proposed for wireless infrastructure-less networks. The main aim of the study of power aware protocol is to help the new researchers and application developers to explore an innovative idea for designing more efficient routing protocols.
\end{abstract}

\section{General Terms}

Mobile Adhoc Networks (MANET), Proactive Routing, Reactive Routing, Hybrid Routing etc.

\section{Keywords}

Destination-Sequenced Distance-Vector (DSDV), Wireless Routing Protocol (WRP), Optimized Link State Routing (OLSR), Dynamic Source Routing (DSR), Temporally Ordered Routing Algorithm (TORA) and Ad-Hoc OnDemand Distance Vector (AODV), Dynamic Source Routing (DSR), Temporally Ordered Routing Algorithm (TORA), Ad-Hoc On-Demand Distance Vector (AODV), Hazy Sighted Link State (HSLS), Cluster Based Routing Protocol (CBR), Zone Routing Protocol (ZRP) etc.

\section{INTRODUCTION}

In recent studies people uses the physical networks based on basic Bellman-Ford routing mechanisms, as specified by RIP. In those networks people face the problems like the poor looping, broken links and time dependent nature of the interconnection topology describing the links between the Mobile Hosts. The basic idea of the design is to operate each Mobile Host as a special router, which systematically advertises its view of the interconnection topology with other Mobile Hosts after regular periods of time within the network. It introduces the mobile adhoc networks [1]. MANET Mobile adhoc network is a self-organizing network in which different wireless nodes dynamically communicate with each other without any already available centralized server. In this type of network there is not any available connection of mobile nodes with the base stations. Transmission of data is very important for the communication between two parties. MANET is a kind of network which made possibility of dynamic communication between nodes but it provides limited bandwidth [2], [4]. Each and every node in MANET behaves as router for packet transmission but separate routers are used in wired networks which transmit packets by maintaining proper routing table. When one node sends information to a different node, a collection of nodes is also employed in between, wherever information is send out totally different hop that's why they are conjointly known as multi-hop, wireless distributed network.In MANETS Nodes can act both as hosts and routers. Bandwidth is also constrained, Different links have different capacity. All the operations performed on MANETs are also in energy constraints and frequent routing updates are also available in MANETs [3]. The behaviour of mobile infrastructure-less network totally depends on the type of routing protocol chosen for the network. There were several techniques or protocols available in recent studies which can be implemented as routing scheme called as traditional Protocols. All these traditional protocols and techniques performed with decreased efficiency that was of no use. Then many proactive, reactive and hybrid protocols were introduced in MANETs .Still energy efficiency was a big problem during the use of proactive, reactive and hybrid protocols so energy economical routing is necessary customary for MANETs. Mobile nodes supercharged by batteries with limited capability in extent [4]. If mobile node in network breakdown it entirely affects the node itself and affects its ability to forward packets on behalf of others so the network period also extends. Some studies classify varied energy-efficient routing mechanisms introduced for wireless infrastructure-less networks. When mobile node sends or receives packets it consumes its battery power. It also stays idle while listening to the wireless medium for any potential communication requests from completely different nodes. Therefore, energyefficient routing protocols minimize either communication energy required to transmit and receive data packets or the energy throughout inactive periods. The transmission power management approach is extended to figure out the simplest routing path that minimizes the complete transmission energy required to deliver data packets to the destination [5]. Another necessary approach to optimizing active communication energy is load distribution approach [6]. Whereas the primary focus of the upper than two approaches is to attenuate energy consumption of individual nodes, the foremost goal of the load distribution technique is to balance the energy usage among the nodes and to maximise the network period by avoiding over-utilized nodes once selecting a routing path [5],[6]. A simple wireless infrastructure-less network is depicted in Fig.1.square shapes presents the mobile nodes which can be connected to any of other mobile nodes through wireless link. Bi-directional arrow shows the wireless link between the nodes in an infrastructure-less network [2]. 




Fig.1: Example of Infrastructure-less Network

\section{RELATED WORK}

In recent studies routing used for wireless adhoc networks was totally based on choosing appropriate route and then maintaining it. All these type of routing is fully dependent on shortest path algorithms. Types of routings are given below in fig 2 [2]:

\subsection{Proactive Energy Based Routing:}

In wired networks the routing table is made by using linkstate algorithms. While using link-state routing a list of next hop, all the destinations, and the number of hops to each destination is maintained by every node to send packets successfully. In wireless network table-driven proactive routing is used, with table driven routing each node sends and receives up to date routing information to every other node in the network [7]. If any change occurs in the network each node update its routing table and transfer the updates to its neighboring nodes. Therefore, in proactive routing when a packet

Needs to be forwarded the route is already known and can be immediately used so in that case not much battery power is consumed for selecting route from multiple available routes. Destination-Sequenced Distance-Vector (DSDV), Wireless Routing Protocol (WRP), Optimized Link State Routing (OLSR) all are proactive routing protocols [7], [8].

\subsection{Reactive Energy Based Routing:}

Reactive based routing is also known as on-demand routing. In this type of routing for route discovery sender mobile node sends route requests to its neighboring nodes then these neighbor nodes forwards route request to their neighbors and so on. When destination node receives the route request it reverts back with an acknowledgement. After route is established successfully sender sends the packets to the destination node. Routes which are previously used stored in the route cache for using available routes in future. Route maintenance is also done during establishment of route [7]. Main aim of on-demand routing is to discover and maintain only needed routes In comparison to table-driven routing protocols, not all updated information about routes are stored at every node [8]. Reactive routing avoids the cost of maintaining routes that are of no use and not misuse the battery power to find unnecessary routes. Dynamic Source Routing (DSR), Temporally Ordered Routing Algorithm (TORA) and Ad-Hoc On-Demand Distance Vector (AODV) are on-demand routing protocols [7], [9].

\subsection{Hybrid Energy Based Routing:}

Hybrid energy based routing is combination both reactive and proactive routing protocols which helps to remove the reduced control traffic overhead from proactive systems meanwhile decreasing the route discovery delays of reactive systems by maintaining routing table. There are few protocols like Hazy Sighted Link State (HSLS), Cluster Based Routing Protocol (CBR) and Zone Routing Protocol (ZRP) etc are called hybrid protocols [7].

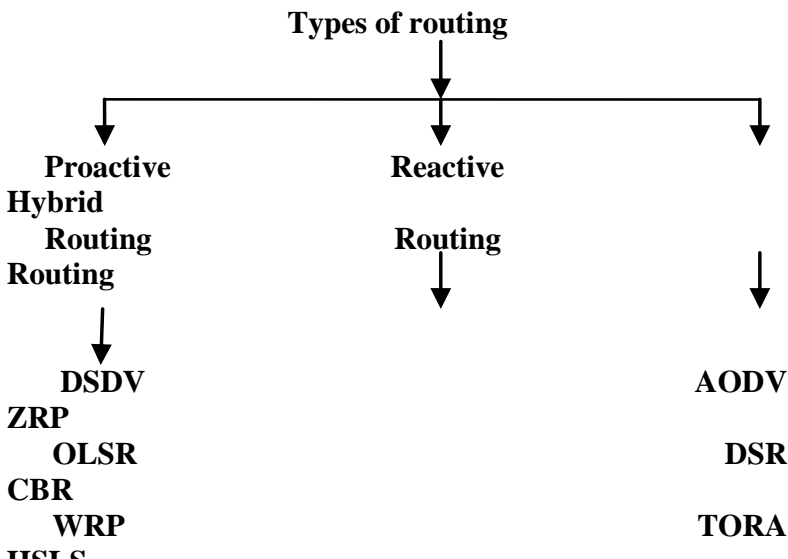

Fig 2: Types of routing

\section{ROUTING PROTOCOLS}

Routing protocols provides best possible route from source to destination. When appropriate route is selected successfully then packet has to go through various in-between mobile nodes. Number of protocols is available for selecting route from source to destination. These protocols may be proactive, reactive or hybrid protocols.

\subsection{DSDV Protocol:}

The DSDV (destination-Sequenced Distance-Vector) protocol works on the basis of Bellman-Ford algorithm to find the shortest number of hops from source to the destination [10]. Each and every DSDV node maintains a routing table which stores all the destinations, number of hops, next hop addresses and sequence numbers [12].

\subsection{OLSR Protocol:}

The OLSR (optimized Link State Routing) is a point to point protocol it is based on the link state packet forwarding mechanism. OLSR optimizes the pure link in two ways. First by decreasing the size of the control packets and second is by decreasing the number of links which are used for forwarding Link state packets [7].

\subsection{WRP Protocol:}

The WRP (Wireless Routing Protocol) It is based distance vector path finding algorithms. WRP maintains the information about next hop and second to the last hop so that counting problem will not arrive. It finds the shortest path from available paths. Each node is sends updates to every other node regularly [7], [10].

\subsection{DSR Protocol:}

The DSR (Dynamic source routing protocol works in two phases route discovery and route maintenance. These phases are used when they are on demand. In route discovery whole 
network is flooded with route requests if appropriate route is not available [11]. Source routing is also implemented in case if complete route to the destination is needed. These routes are stored in cache .After route is established route maintenance is done so that packets cannot be lost [12].

\subsection{AODV Protocol:}

The AODV (adhoc on demand distance vector protocol).It is simple enhancement over DSR protocol. It is based on distance vector routing. In AODV protocol routes are searched only when they are in demand and every node maintains its own temporary routing table. AODV works mainly on the basis of Route Request (RREQ), Route Replies (RREP) and Route Errors (RERR) [10].

\subsection{TORA Protocol:}

The TORA (temporally ordered routing algorithm) is reactive type of protocol. It contains of destination-oriented directed acyclic graph (DAG).It also uses the concept of link reversal in case If links get failed [7].

Besides above explained protocols there are other protocols like ZRP (Zone Routing protocol), CBR (Cluster Based Routing Protocol), and HSLS(Hazy Sighted Link State) etc all are hybrid protocols means that they are combination of both proactive and reactive routing Protocols [11].

\section{ENERGY EFFICIENT ROUTING PROTOCOL}

In adhoc network or any other wireless network battery power is major necessity. The technology friendly devices that operates on battery power helps to increase energy efficiently by decreasing the energy they consume, also maintains the performance as per expectance. Power consumption is not only the measure for energy efficiency. Energy efficiency can also be measured by noting the time for which network performs very well called as network lifetime. Sometimes when routes with lowest energy are followed .Through these routes more traffic can flow but it adversely affects all the nodes present in the network. These nodes get exhausted in very short time so in that case network cannot give good results due to failure of network nodes. For better energy efficiency power consumed by every node should be in balanced amount simultaneously network lifetime should be maximum so all the routes and nodes get balanced globally [13].

There are many types of routing algorithms. First is broadcasting. In broadcasting if any rout gets failed then it broadcasts message to other nodes so that new route get formulated simply. The second type is multicasting in routing protocols. During multicasting one group of nodes can communicate with multiple other groups of nodes. Last type is uni-cast routing in which only one to one communication take place. In wireless network nodes can be failed. It is hard to save energy while broadcasting as re-routing is required during node failure. During multicasting saving energy is same big challenge to achieve as in broadcasting. In uni-cast saving energy completely depends on status of link [14].

In adhoc networks energy is limiting value all other factors totally depends on energy. It is necessary to use energy in proper way. Nodes behavior depends on few characteristics they are:

Firstly energy of nodes completely depends on battery with limited power supply. Secondly there is chance of failure in routes because nodes are mobile means that they can move without any central control. Thirdly bandwidth of wireless network is very limited in comparison to wired network. Bandwidth is not constant it varies time to time. Wireless network have very low bandwidth which adversely affect the network. Sometime above characteristics creates many problems like node failure, route failure etc in network.

To get rid of above problems only one solution is possible that is to design energy efficient protocols. Making protocol energy efficient is mostly done in reactive protocols as they are more energy efficient than proactive protocols. The energy efficient protocols which are already introduced still have many drawbacks. Flooding in proper sense in reactive protocol can also help in achieving energy efficiency. If effective metrics such as cost, per node energy and battery level are used for route selection it will also save energy properly [15], [16].

DIFFERENT ENERGY BASED METRICS: There are many energy efficient protocols which help in decreasing the power consumption by using different metrics in routing. These routing metrics are used in computing routing table. If a routing protocol use the routing metrics it can easily maintains energy efficiency in its packet forwarding. In routing metrics protocol either tries to route the packets through the maximum energy path or tries to minimize the end to end transmission energy of packets. In first case the total energy consumed to send the data along the route is find out so in this overall transmission power consumed per packet is reduced but it does not have any impact on the lifetime of each node. In second case end to end transmission energy is minimized only if nodes adjust their transmission power levels so this process helps in maintaining good network lifetime [17].

\section{CONCLUSION}

There is not any single protocol which can use battery power efficiently. Energy efficient protocols which are developed already lacks in some criteria like In some protocols overall transmission power consumed by every packet cannot be reduced and in some protocols nodes cannot adjust their transmission power levels. Different protocols behave differently in adhoc networks. Performance of the routing protocols varies on the basis of variation in network parameters like nodes are mobile they can move in uncontrolled way, their behaviour also depends on the power supplied by battery and variation in low bandwidth. There should be some explicit protocol which can offer the most effective performance in each and every case topology. Therefore protocol with adjustable power levels per node, which will provide better network lifetime, is required. Protocol should be picked carefully which will be adapted in every topology and provides better energy efficiency. This review will help the researchers to pick good energy efficient protocol and also helps them to introduce new energy efficient protocol.

\section{REFERENCES}

[1] Charles E. Perkins and Pravin Bhagwat, 1994.Highly Dynamic Distance Destination-Sequenced Vector Routing. In Proceedings of the ACM SIGCOMM, Vol. 24, No. 4, October, 1994, pp.234-244.

[2] Anuj K. Gupta, Harsh Sadawarti, and Anil K. Verma, 2011. Review of Various Routing Protocols for MANETs. International Journal of Information and 
Electronics Engineering, Vol. 1, No. 3, November 2011, pp.251-259.

[3] Chintan Patel, Prof. Vyomal N. Pandya , Prof. Milind Shah , 2013. Survey of Reactive Routing Protocols for MANET. IOSR Journal of Electronics and Communication Engineering, Vol. 5, No. 3, April, 2013, pp.84-92.

[4] Shiva Prakash, J.P. Saini, Ajeet kumar Gautam, S.C. Gupta, 2013.Literature Review and General Consideration of Energy Efficient Routing Protocols in MANETs. International Journal of Advanced Research in Computer Science and Software Engineering, Vol. 3, No. 12, December 2013, pp. 198-206.

[5] V. Kawadia and P.R. Kumar, 2003. Power Control and Clustering in Ad hoc Networks. Wireless Communications, IEEE INFOCOM, Vol. 12, No.1, 2003, pp.3-11

[6] Radhika D. Joshi and Priti P. rege, 2008. Distributed Energy Efficient Routing in Ad-hoc Networks. IEEE, Fourth International Conference on Wireless Communication and Sensor Networks (WCSN 2008), 27-29 Dec., 2008, pp.16-21.

[7] Prashant Mohapatra and Srikanth V. Krishnamurthy," AD HOC NETWORKS Technologies and Protocols", Springer.

[8] N. S. Yadav and R.P. Yadav "Performance Comparison and Analysis of Table- Driven and On- Demand Routing Protocols for Mobile Adhoc Networks," "International Journal of Information Technology", vol.4, no. 2, pp 101-109, 2007

[9] Y. Hu, A. Perrig and D. Johnson, Ariadne: A Secure Ondemand Routing Protocol for Ad Hoc Networks, "in Proceedings of ACM MOBICOM'02", 2002.

[10] A. Boukerche, B. Turgut, N. Aydin, M. Z. Ahmad, L. Bölöni, and D.Turgut, "Routing protocols in ad hoc networks: A survey," "Elsevier Computer Networks", 55 (2011) 3032-3080.

[11] E. M. Royer and Chai-Keong Toh, "A Review of Current Routing Protocols for Ad Hoc Mobile Wireless Networks"

[12] B. Divecha, A. Abraham, C. Grosan, and S. Sanyal, "Analysis of Dynamic Source Routing and DestinationSequenced Distance-Vector Protocols for Different Mobility models," in Proc. of First Asia International Conference on Modelling \& Simulation, Phuket, Thailand, 27-30 March, 2007, pp. 224-229.

[13] Jangsu Lee, Seunghwan Yoo, and Sungchun Kim, "Energy aware Routing in Location based Ad-hoc Networks," Proceedings of the 4th International Symposium on Communications, Control and Signal Processing, ISCCSP 2010, Limassol, Cyprus, 3-5 March 2010.

[14] Xiaoying Zhang, Thomas Kunz, Li Li and Oliver Yang, "An Energy-efficient Broadcast Protocol in MANETs," Communications Networks and Services Research Conference, Proceedings of the 2010 8th Annual Communication Networks and Services Research Conference, Pages: 199-206 SBN: 978-0-7695-4041-2.

[15] Juan A. Sanchez and Pedro M. Ruiz, “ LEMA: Localized Energy-Efficient Multicast Algorithm based on Geographic Routing," in Proceedings. Of 2006 31st IEEE Conference on Local Computer Networks in 2006.

[16] Dahai Du and Huagang Xiong, "A Location aided Energy-Efficient Routing Protocol for Ad-hoc Networks," in wireless and optical communications conference (WOCC), 2010 19th annual.

[17] S. Shakkottai, T. S. Rappaport, and P. C. Karlsson, "Cross-layer design for wireless networks," IEEE Commun. Mag., vol. 41, no. 10, pp. 74_49, Oct. 2003. 